\title{
Transplante autógeno em dentes posteriores: Revisão Integrativa
}

\author{
Autogenous transplantation in posterior teeth: Integrative Review \\ Trasplante autógeno en dientes posteriores: revisión integradora
}

Recebido: 25/02/2021 | Revisado: 04/03/2021 | Aceito: 07/03/2021 | Publicado: 15/03/2021

\author{
Alessandro Ítalo Cruz \\ ORCID: https://orcid.org/0000-0003-4679-7302 \\ Centro Universitário Tiradentes, Brasil \\ E-mail: aleitalocruz@gmail.com \\ Anna Clara Rodrigues Bezerra \\ ORCID: https://orcid.org/0000-0003-2636-5932 \\ Centro Universitário Tiradentes, Brasil \\ E-mail: Nnaclara_rodrigues@hotmail.com \\ Antonio Carlos Oliveira de Araujo \\ ORCID: https://orcid.org/0000-0001-6950-8070 \\ Centro Universitário Tiradentes, Brasil \\ E-mail: Antoniocarloos296@gmail.com \\ Dayse Andrade Romão \\ ORCID: https://orcid.org/0000-0002-7884-1657 \\ Centro Universitário Tiradentes, Brasil \\ E-mail: Dayseromao@gmail.com \\ Estéfane Carvalho do Nascimento \\ ORCID: https://orcid.org/0000-0002-4372-9657 \\ Centro Universitário Tiradentes, Brasil \\ E-mail: Estefane.carvalho@outlook.com \\ Luan Marcel Gonçalves Mélo \\ ORCID: https://orcid.org/0000-0002-8297-3054 \\ Centro Universitário Tiradentes, Brasil \\ E-mail: Luan.marcel@souunit.com.br \\ Mariana Alencar Nemezio \\ ORCID: https://orcid.org/0000-0003-1852-7882 \\ Centro Universitário Tiradentes, Brasil \\ E-mail: Marianaalne@hotmail.com \\ Wanderson Thalles de Souza Braga \\ ORCID: https://orcid.org/0000-0003-3617-3950 \\ Centro Universitário Tiradentes, Brasil \\ E-mail: Wanderson.thalles@souunit.com.br \\ Wirla Jesus dos Santos \\ ORCID: https://orcid.org/0000-0002-8921-8596 \\ Centro Universitário Tiradentes, Brasil \\ E-mail:wirlajesus@gmail.com
}

\section{Resumo}

O transplante dental autógeno pode ser definido como o movimento cirúrgico de um dente incluso ou erupcionado de um local para outro, num mesmo indivíduo, em alvéolos de dentes recém-extraídos ou preparados cirurgicamente. Objetivo: fazer uma análise na literatura sobre os benefícios do transplante dentário autógeno em dentes posteriores como alternativa terapêutica para reabilitação de pacientes. Método: Trata-se de uma revisão integrativa realizada entre os meses de fevereiro a abril de 2020 a partir de aquisição de dados científicos das plataformas SciELO, LILACS, Medline, Pubmed. Essa revisão é referente ao período de 2010 até 2020 nos idiomas: português e inglês. Utilizou-se para a realização da busca individual, os descritores: Autotransplante; Reabilitação Bucal; Autógeno, "Autotransplantation", "Oral rehabilitation", "Autogenous" além do cruzamento entre eles utilizando o operador booleano "AND”. Resultado: após a realização de uma busca na literatura foram encontrados oito artigos relacionados com o Transplante dentário autógeno de dentes posteriores. Conclusão: o transplante dental autógeno (TDA) pode ser considerado uma alternativa viável, os índices de sucesso é grande e permite devolver as características estéticas e funcionais ao paciente.

Palavras-chave: Autotransplante; Reabilitação bucal; Autógeno.

\footnotetext{
Abstract

Autogenous dental transplantation can be defined as the surgical movement of an embedded or erupted tooth from one location to another, in the same individual, in alveoli of newly extracted or surgically prepared teeth. Objective: To exemplify the success of autogenous dental transplantation and discuss the feasibility of using this technique as a therapeutic alternative for the rehabilitation of children and adolescents. Method: This is an integrative review on
} 
autogenous posterior teeth dental transplantation carried out between February and April 2020, based on the acquisition of scientific data from SciELO, LILACS, Medline, Pubmed platforms. This review refers to the period from 2010 to 2020 in the languages: Portuguese, English. The following descriptors were used to perform the individual search: Autotransplantation; oral rehabilitation; Autogenous, in addition to the crossing between them using the Boolean operator "AND”. Result: after conducting a literature search, eight articles related to autogenous posterior teeth dental transplantation were found. Conclusion: autogenous dental transplantation (ADT) can be considered a viable alternative, the success rate is high and allows the patient to return the aesthetic and functional characteristics.

Keywords: Autotransplantation; Oral rehabilitation; Autogenous.

\section{Resumen}

El trasplante dental autógeno se puede definir como el movimiento quirúrgico de un diente incrustado o erupcionado de un lugar a otro, en el mismo individuo, en los alvéolos de dientes recién extraídos o preparados quirúrgicamente. Objetivo: realizar un análisis en la literatura sobre los beneficios del trasplante dentario autógeno en dientes posteriores como alternativa terapéutica para la rehabilitación de pacientes. Método: Se trata de una revisión integradora realizada entre febrero y abril de 2020, basada en la adquisición de datos científicos de las plataformas SciELO, LILACS, Medline, Pubmed. Esta revisión se refiere al período de 2010 a 2020 en los idiomas: portugués, inglés. Se utilizaron los siguientes descriptores para realizar la búsqueda individual: Autotransplantation; Rehabilitación oral; Autógeno, “Autotrasplante", "Rehabilitación oral", "Autógeno" además del cruce entre ellos mediante el operador booleano "Y”. Resultado: luego de realizar una búsqueda bibliográfica, se encontraron ocho artículos relacionados con el trasplante dental autógeno de dientes posteriores. Conclusión: el trasplante dentario autógeno (TPA) puede considerarse una alternativa viable, las tasas de éxito son altas y permiten al paciente devolver las características estéticas y funcionales. Palabras clave: Autotrasplante; Rehabilitación oral; Autógeno.

\section{Introdução}

O transplante dental autógeno (TDA) é a transferência de um dente com vitalidade ou tratado endodonticamente do seu local de origem para outro sítio, sendo necessário que esse elemento pertença ao mesmo indivíduo para ser considerado autógeno (Nimčenko et al., 2013). Esses transplantes são realizados há mais de 40 anos nos países escandinavos, permitindo uma elevada taxa de sucesso na população destes países, sendo procedimentos rotineiros e com protocolos bem estabelecidos nos serviços odontológicos (Santos et al., 2013).

Os primeiros transplantes dentários foram realizados no antigo Egito onde escravos e estrangeiros eram obrigados a ceder seus dentes aos seus senhores. Em 1564, Ambroise Pare foi o primeiro a relatar com sucesso o transplante dental. Apfel e Miller em (1950) foram os primeiros a descrever transplantes na literatura científica. No Brasil, Clovis Marzola se destacou pela sua produção técnica e científica sobre os transplantes dentais (Kumar et al., 2012; Yoshino et al., 2012; Santos et al., 2013).

Atualmente, existem duas técnicas cirúrgicas distintas, quando a cirurgia é realizada em uma única sessão e o dente transplantado em um período superior a quatro horas pós-remoção é a técnica clássica. Já a técnica composta é realizada em duas sessões com intervalo de 15 dias, onde na primeira sessão o alvéolo receptor é preparado para receber o dente transplantado em uma segunda sessão (Costa et al., 2013). Ambas as técnicas são utilizadas para o transplante autógeno do terceiro molar para o lugar do primeiro ou segundo molar (Santos et al., 2013).

$\mathrm{Na}$ literatura são observados transplantes de incisivos traumatizados à molares (Costa et al., 2013). O TAD sendo uma opção viável para a substituição de um elemento dentário comprometido, mantendo a função proprioceptiva do dente, impedindo a migração dental, preservando a altura do osso alveolar e estimulando o desenvolvimento dento facial natural, estabelecendo uma estética local (Tirali et al., 2013). Porém, alguns requisitos devem ser observados para um sucesso no procedimento, como: os pacientes precisam ter boa saúde geral, o elemento a ser transplantado tem de ser compatível com o tamanho do alvéolo, além disso tem que estar em condições aceitáveis (Mikami, et al., 2014).

Desta forma, o TAD é um tratamento de baixo custo, pois não há necessidade de gastos com implantes dentários ou com tratamentos protéticos, mesmo com a existência de complicações, como anquilose e perda do elemento transplantado (Costa et al., 2013). Assim, este trabalho tem como objetivo fazer uma análise na literatura sobre os benefícios do transplante dentário autógeno de dentes posteriores como alternativa terapêutica para reabilitação de pacientes. 


\section{Metodologia}

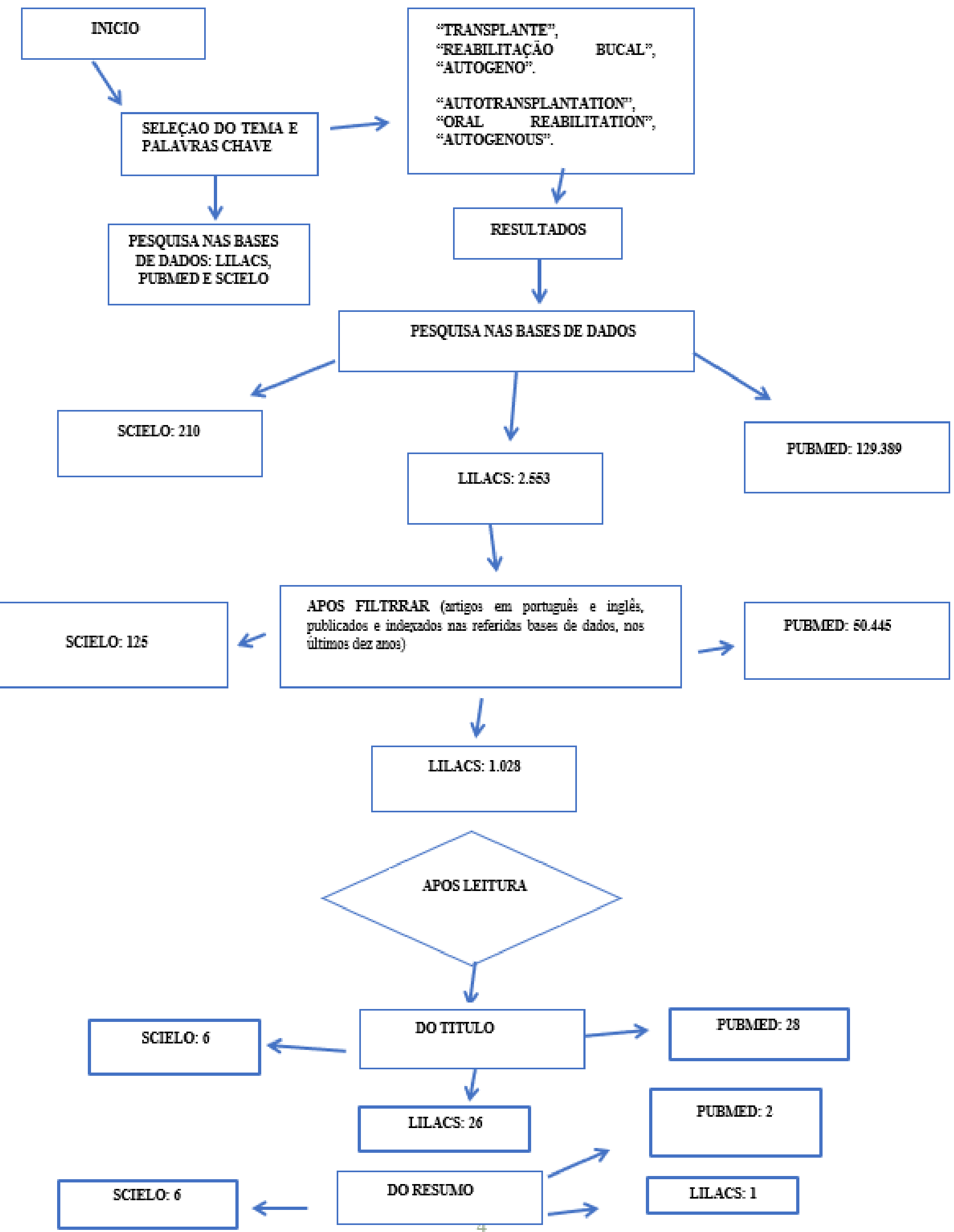


Neste estudo, foi realizada uma revisão integrativa da literatura, definida como aquela em que as pesquisas já publicadas são sintetizadas e geram conclusões sobre o tema em estudo. A elaboração da revisão integrativa compreende seis etapas: identificação do tema e seleção da hipótese ou questão de pesquisa para a elaboração da revisão integrativa, estabelecimento de critérios para inclusão e exclusão de estudos/ amostragem ou busca na literatura, definição das informações a serem extraídas dos estudos selecionados/categorização dos estudos, avaliação dos estudos incluídos na revisão integrativa, interpretação dos resultados, apresentação da revisão/síntese do conhecimento (Mendes et al., 2008).

A questão condutora desta pesquisa foi: quais são os benefícios do transplante dentário autógeno em dentes posteriores como alternativa terapêutica?

Para a busca dos artigos foram utilizadas as seguintes bases de dados: Literatura Latino-Americana e do Caribe em Ciências da Saúde (Lilacs), National Library of Medicine, EUA (Pubmed) e o repositório Scientific Electronic Library Online (Scielo).

Para o levantamento dos artigos foram utilizados como descritores, "Transplante", "Reabilitação Bucal", "Autógeno". “Autotransplantation”, “Oral rehabilitation”, “Autogenous”. Realizou-se a busca pelos descritores individualmente. Em seguida, foram realizados os cruzamentos utilizando o operador boleano and. Posteriormente, os três descritores foram cruzados em conjunto. Os critérios de inclusão para a seleção da amostra foram: artigos publicados em português e inglês, publicados e indexados nas referidas bases de dados, nos últimos dez anos (2010 a 2020), e que retratassem a temática em estudo. Para critério de exclusão foram: Dissertações, monografias e teses. Os Quadros 1, 2 e 3 evidenciam a estratégia de busca utilizada.

Os Quadros 1,2 e 3 evidenciam as estratégias de busca utilizada.

Quadros de Publicação:

Quadro 1: Publicações encontradas entre os anos de 2010 a 2020 segundo a base de dados Scielo.

\begin{tabular}{|c|c|c|c|c|}
\hline Descritor & Total de publicações & $\begin{array}{c}\text { Publicações } \\
\text { filtradas }\end{array}$ & $\begin{array}{c}\text { Após leitura do } \\
\text { título }\end{array}$ & $\begin{array}{c}\text { Após leitura do } \\
\text { resumo }\end{array}$ \\
\hline Autotransplante & 46 & 6 & 3 & 3 \\
\hline Reabilitação Bucal & 58 & 47 & 1 & 0 \\
\hline $\begin{array}{c}\text { Autógeno } \\
\text { Reabilitação Bucal }\end{array}$ & 162 & 68 & 0 & 1 \\
\hline $\begin{array}{c}\text { Autotransplante and } \\
\text { Autógeno }\end{array}$ & 3 & 1 & 0 & 0 \\
\hline $\begin{array}{c}\text { Reabilitação bucal } \\
\text { and Autógeno }\end{array}$ & 1 & 2 & 0 & 0 \\
\hline $\begin{array}{c}\text { Autotransplante and } \\
\text { Reabilitação bucal } \\
\text { and Autógeno }\end{array}$ & 1 & 1 & & 0 \\
\hline
\end{tabular}

Fonte: Autores. 
Quadro 2: Publicações encontradas entre os anos de 2010 a 2020 segundo a base de dados Lilacs.

\begin{tabular}{|c|c|c|c|c|}
\hline Descritor & Total de publicações & $\begin{array}{c}\text { Publicações } \\
\text { filtradas }\end{array}$ & $\begin{array}{c}\text { Após leitura do } \\
\text { título }\end{array}$ & $\begin{array}{c}\text { Após leitura do } \\
\text { resumo }\end{array}$ \\
\hline Autotransplante & 996 & 293 & 4 & 0 \\
\hline Reabilitação Bucal & 786 & 400 & 4 & 0 \\
\hline Autógeno & 629 & 285 & 12 & 0 \\
\hline $\begin{array}{c}\text { Autotransplante and } \\
\text { Reabilitação Bucal }\end{array}$ & 45 & 11 & 1 & 0 \\
\hline $\begin{array}{c}\text { Autotransplante and } \\
\text { Autógeno }\end{array}$ & 50 & 10 & & 0 \\
\hline $\begin{array}{c}\text { Reabilitação bucal } \\
\text { and Autógeno }\end{array}$ & 39 & 24 & & 0 \\
\hline $\begin{array}{c}\text { Autotransplante and } \\
\text { Reabilitação bucal } \\
\text { and Autógeno }\end{array}$ & 8 & 5 & & 0 \\
\hline
\end{tabular}

Fonte: Autores

Quadro 3: Publicações encontradas entre os anos de 2010 a 2020 segundo a base de dados Pubmed.

\begin{tabular}{|c|c|c|c|c|}
\hline Descritor & $\begin{array}{c}\text { Total de } \\
\text { publicações }\end{array}$ & $\begin{array}{c}\text { Publicações } \\
\text { filtradas }\end{array}$ & $\begin{array}{c}\text { Após leitura do } \\
\text { título }\end{array}$ & $\begin{array}{c}\text { Após leitura do } \\
\text { resumo }\end{array}$ \\
\hline Autotransplante & 79,202 & 25,524 & 15 & 0 \\
\hline Reabilitação Bucal & 30,613 & 15,178 & 5 & 0 \\
\hline Autógeno & 15,668 & 8,660 & 28 & 0 \\
\hline $\begin{array}{c}\text { Autotransplante and } \\
\text { Reabilitação Bucal }\end{array}$ & 291 & 119 & 10 & 0 \\
\hline $\begin{array}{c}\text { Autotransplante and } \\
\text { Autógeno }\end{array}$ & 3,180 & 736 & 0 & 0 \\
\hline $\begin{array}{c}\text { Reabilitação bucal and } \\
\text { Autógeno }\end{array}$ & 366 & 197 & & 0 \\
\hline $\begin{array}{c}\text { Autotransplante and } \\
\text { Reabilitação bucal and } \\
\text { Autógeno }\end{array}$ & 69 & & & 0 \\
\hline
\end{tabular}

Fonte: Autores.

As estratégias utilizadas para o levantamento dos artigos foram adaptadas para cada uma das bases de dados, de acordo com suas especificidades de acesso, sendo guiadas pela pergunta norteadora e critérios de inclusão. Para a seleção dos artigos foram lidos todos os titulos e selecionados aqueles que tinham relação com o objetivo do estudo. Em seguida foram analisados os resumos e elegidos para a leitura do artigo na integra aqueles que estavam relacionados com a temática em estudo.

Em suma foram lidos 42 artigos e escolhidos 8 os quais respondiam à questão condutora do estudo e se encaixavam nos critérios de inclusão da Revisão Integradora. A realização dos levantamentos bibliográficos ocorreu nos meses de Março e abril 
de 2020. Os artigos encontrados foram enumerados conforme a ordem de localização, identificados e apresentados conforme as normas de referência bibliográfica.

\section{Resultados}

Nesta revisão integrativa, foram abordados no Quadro 4, 8 artigos científicos, os quais obedeceram aos requisitos de inclusão anteriormente definidos. As informações do Quadro 4 tratam de um breve resumo das características dos estudos incluídos.

Quadro 5. Apresentação das características dos artigos incluídos na Revisão Integrativa.

\begin{tabular}{|c|c|c|c|c|}
\hline Título & Autores & Ano & Delineamento & Desfecho \\
\hline $\begin{array}{l}\text { Transplante dental } \\
\text { bilateral: relato de } \\
\text { caso clínico com } \\
\text { acompanhamento de } \\
9 \text { anos }\end{array}$ & Jodas et al. & 2012 & Relato de caso & $\begin{array}{l}\text { Foi realizado um autotransplantdentário } \\
\text { bilateral em um adulto jovem que apresentou } \\
\text { elementos com as raízes residuais, as } \\
\text { exodontias foram realizadas devido lesões de } \\
\text { cáries extensas. }\end{array}$ \\
\hline $\begin{array}{l}\text { Autotransplante } \\
\text { Dentário: Uma opção } \\
\text { reabilitadora e viável } \\
\text { ao SUS }\end{array}$ & Rebouças et al. & 2015 & Revisão de literatura & $\begin{array}{l}\text { Pacientes que perderam os dentes devido a } \\
\text { cáries ou fraturas em indivíduos jovens revela- } \\
\text { se um desafio, sobretudo para os indivíduos } \\
\text { economicamente desfavorecidos, pois as } \\
\text { formas convencionais de reabilitação } \\
\text { apresentam alto custo. Um tratamento } \\
\text { alternativo para a reposição dos dentes } \\
\text { comprometidos é o transplante dentário. } \\
\begin{array}{l}\text { Demonstrando-se como uma opção viável, } \\
\text { principalmente por ser uma técnica } \\
\text { relativamente simples. }\end{array}\end{array}$ \\
\hline $\begin{array}{l}\text { Autotransplantation } \\
\text { of premolars to the } \\
\text { maxillary incisor } \\
\text { region: a long-term } \\
\text { follow-up of } 12-22 \\
\text { years }\end{array}$ & $\begin{array}{l}\text { Stange; } \\
\text { Lindsten; } \\
\text { Bjerklin. }\end{array}$ & 2016 & Relato de caso & $\begin{array}{l}\text { O autotransplante tem a vantagem de preservar } \\
\text { e estimular o crescimento ósseo contínuo. } \\
\text { Além disso, o dente ausente é substituído sem } \\
\text { envolver os dentes vizinhos }\end{array}$ \\
\hline $\begin{array}{l}\text { Autotransplante } \\
\text { dentário: uma } \\
\text { alternativa viável para } \\
\text { a reabilitação oral }\end{array}$ & Duarte et al. & 2017 & Relato de caso & $\begin{array}{l}\text { O autotransplante dentário é considerado uma } \\
\text { excelente alternativa para reabilitação de } \\
\text { perdas dentárias em adolescentes. Esta técnica } \\
\text { consiste no reposicionamento de um dente } \\
\text { autógeno no local da ausência dentária, por } \\
\text { meio da exodontia do elemento indicado ou } \\
\text { preparação de um alvéolo cirúrgico. }\end{array}$ \\
\hline $\begin{array}{l}\text { Two-stage technique } \\
\text { in third molar } \\
\text { autotransplantation: } \\
\text { case report }\end{array}$ & $\begin{array}{l}\text { Pinto Junior et } \\
\text { al. }\end{array}$ & 2018 & Relato de caso & $\begin{array}{l}\text { O autotransplante dentário é uma opção para o } \\
\text { tratamento de pacientes com perda dentária } \\
\text { precoce. Permitindo que o dente } \\
\text { comprometido ou ausente seja substituído por } \\
\text { um dente transplantado, devolvendo a } \\
\text { funcionalidade e estética. }\end{array}$ \\
\hline $\begin{array}{l}\text { Autotransplantation } \\
\text { of teeth with complete } \\
\text { rhizogenesis: a } \\
\text { literature review }\end{array}$ & Ribeiro et al. & 2019 & Revisão de literatura & $\begin{array}{l}\text { Estudos de autotransplante realizados em } \\
\text { pacientes com rizogênese completa, a técnica } \\
\text { tem se } \\
\text { mostrado uma opção viável. }\end{array}$ \\
\hline $\begin{array}{l}\text { Transplante dentário } \\
\text { autógeno: revisão de } \\
\text { literatura e relato de } \\
\text { caso clínico }\end{array}$ & Warmeling et al. & 2019 & $\begin{array}{l}\text { Relato de caso e } \\
\text { revisão de literatura }\end{array}$ & $\begin{array}{l}\text { Observou o autotransplante resultando em um } \\
\text { bom prognóstico e opção viável para a } \\
\text { substituição de molares ausentes ou com cáries } \\
\text { extensas. }\end{array}$ \\
\hline $\begin{array}{lr}\text { Transplante } & \text { Dental } \\
\text { Autógeno } & \text { como } \\
\text { Alternativa } & \text { à } \\
\text { Reabilitação Oral } & \\
\end{array}$ & $\begin{array}{l}\text { Da Silva } \\
\text { Fonseca et al. }\end{array}$ & 2019 & Relato de caso & $\begin{array}{l}\text { Foi realizado autotransplante após meses de } \\
\text { acompanhamento observou-se vitalidade e } \\
\text { ausência de bolsas periodontais no elemento } \\
\text { transplantado }\end{array}$ \\
\hline
\end{tabular}

Fonte: Autores. 


\section{Discussão}

Os primeiros molares permanentes são os principais dentes perdidos precocemente (Batista \& Rihs \& Sousa, 2012) pelo acometimento de lesões de cárie extensa, devido ao fato de irromperem mais cedo na cavidade oral e por ser erroneamente visto como dente decíduo (Costa et al., 2013). A ausência desse dente leva a graves deficiências na mastigação de alimentos, implicando também uma sobrecarga não fisiológica sobre os dentes restantes. Esta ausência de dentes posteriores poderá ainda ser responsável por problemas articulares, dificultando a realização de reabilitações posteriores (Lacerda et al., 2013).

Para reabilitar um paciente que teve uma perda dentária, as principais opções são as próteses sobre implantes, as próteses fixas ou removíveis (Rebouças et al., 2015), porém o autotransplante dentário vem sendo uma ótima alternativa para reabilitação oral em pacientes jovens quando o tratamento protético for inviável por motivos socioeconômicos (Peixoto \& Melo \& Santos., 2013). O transplante dentário (TD) é uma opção de tratamento para reposição de elementos dentários quando há um dente doador viável, podendo ser indicado em casos de dentes inclusos, agenesia dental e perda prematura de dentes devido a trauma, cárie ou doença periodontal, feito através da substituição de um elemento perdido ou ausente por um dente elemento (Peixoto et al., 2013; da Silva Fonseca et al., 2019). Este procedimento apresenta vantagens, evitando alterações no desenvolvimento da maxila e da mandíbula, com um tratamento conservador, possibilitando o desenvolvimento do osso alveolar na área receptora, podendo realizar movimentações ortodôntica e recuperação do espaço do dente perdido (Stange \& Lindsten \& Bjerklin., 2015).

O transplante dental pode ser, autógeno, que é quando o doador e o receptor é a mesma pessoa, homógeno, quando a transferência é de um indivíduo para outro, porém da mesma espécie; e heterógeno, realizado entre membros de espécies diferentes. Normalmente o transplante autógeno ocorre do terceiro molar para o lugar do primeiro ou segundo molar; de prémolares para o lugar de incisivos e de caninos impactados para sua posição funcional (Giancristófaro et al., 2009). No caso de transplante de molares, devido as grandes variações o índice de sucesso altera nas seleções de caso (formação radicular incompleta versus formação radicular completa). Nos pré-molares o prognóstico de transplante é favorável especialmente se realizado no estágio em que está ocorrendo o desenvolvimento radicular, incisivos e caninos o prognóstico a longo prazo parece revelar bons resultados (Jodas et al., 2012).

O exame imaginológico, bem como a radiografia periapical, são imprescindíveis na seleção e indicação da cirurgia, principalmente para estabelecer o estágio de formação radicular, o diâmetro mésio-distal do dente a ser transplantado e as possíveis patologias no sítio cirúrgico (da Silva Fonseca et al., 2019). Afim de evitar algum tipo de infecções cruzadas, o pós operatório influência sobre o resultado do procedimento, especialmente quando se refere ao uso de antibióticos e higienização adequada da área manipulada (Duarte et al., 2017).

O comprimento radicular ideal é de $2 / 3$ a 3/4 da raiz formada, com forame apical aberto, para que haja revascularização sem tratamento endodôntico (Atala-acevedo et al., 2017; Warmeling et al., 2019; Da Silva Fonseca et al. 2019). A área receptora deve ter boa estrutura óssea para dar estabilidade e ser livre de quaisquer infecções ou inflamações (Warmeling et al., 2019). No entanto, embora a preferência seja por dentes com forame apical aberto, da Silva Fonseca et al., (2019) afirma que há relatos de sucesso da técnica realizada no implante de dentes com rizogênese completa. De acordo com Jodas et al. (2012), outra técnica de sucesso é luxação e reimplante associado a enxerto de osso bovino com colágeno tipo 1 para estabilizar o dente em sua nova posição.

Em alguns casos, o procedimento é contraindicado: pacientes com menos de 12 anos, visto que o desenvolvimento radicular encontra-se insatisfatório; elemento doador com tamanho superior ao da área receptora; existência de infecções agudas ou perda óssea crônica; pacientes com áreas desdentadas de vários dentes, com grande susceptibilidade à cárie, com má higiene oral e que tenham patologia sistémica que impeça ou condicione a realização da cirurgia (Jodas et al., 2012). Em casos que necessitem realizar odontossecção devido a morfologia radicular complicada (Ravi et al., 2012). 
Antes de realizar o procedimento é necessário comunicar ao paciente algumas complicações: anquilose, reabsorção radicular, necrose pulpar e anquilose (Rebouças et al. 2015). Em casos com formação inferior a 2/3 pode ser traumática, atrapalhar a maturação e consequente desenvolvimento radicular (Kumar et al, 2012). Segundo Warmeling et al., (2019), um dos parâmetros é o suporte ósseo em todas dimensões da área receptora, ausência de infeção aguda, inflamação crônica e presença de tecido queratinizado harmônico para consolidação do dente transplantado.

Entre os fatores que influenciam no sucesso estão: idade do paciente, técnica utilizada no procedimento, anatomia e condições do dente doador (Rebouças et al. 2015). Já de acordo com Ribeiro et al., (2019), as mulheres tem maiores taxas de sucessos pois são mais colaborativas nos tratamentos odontológicos e também apresentam melhores hábitos de higiene oral.

\section{Conclusão}

Existem várias abordagens terapêuticas para reabilitação de perdas dentárias que visam o reestabelecimento da função mastigatória de pacientes. No entanto, o transplante Autógeno de elementos dentários apresenta vários benefícios frente aos procedimentos protéticos. Perante a literatura consultada, o transplante dental autógeno (TDA) pode ser considerado uma alternativa viável, os índices de sucesso é grande e permite devolver as características estéticas e funcionais ao paciente. É de suma importância que o cirugião dentista observe todos os critérios de seleção do elemento dentário tanto como uma boa técnica cirurgica para um sucesso do procedimento.

Os próximos estudos poderiam abordar os casos em que o autotransplante foi realizado e não se obteve taxa de sucesso, bem como salientar as condições desfavoráveis que faz com que o insucesso ocorra.

\section{Referências}

Atala-Acevedo, C., Abarca, J., Martínez-Zapata, M. J., Diaz, J., Olate, S., \& Zaror, C. (2017). Success rate of autotransplantation of teeth with na open apex: systematic review and meta-analysis. Journal of Oral and Maxillofacial Surgery, 75(1), 35-50. 10.1016/j.joms.2016.09.010

Batista, M. J., Rihs, L. B., \& Sousa, M. D. L. R. D. (2012). Risk indicators for tooth loss in adult workers. Brazilian oral research, 26(5), 390-396. $10.1590 / \mathrm{S} 1806-83242012000500003$

Costa, D. R., Muñoz, I. S. S., \& Nicolau, R. A. Nível de sucesso de transplante autógenos de dentes permanentes-Revisão de literatura. Revista Univap. http://www.inicepg.univap.br/cd/INIC_2013/anais/arquivos/0586_0289_01.pdf

Da Silva Fonseca, T., da Silva, R. L. Q., de Andrade Hage, C., \& Ferreira, D. P. (2019). Transplante dental autógeno como alternativa á reabilitação oral. Revista Odontológica do Brasil Central, 28(85). https://doi.org/10.36065/robrac.v28i85.1052

Da Silva, B. R., da Silva Fidalgo, T. K., \& Silva, E. J. N. L. (2019). Autotransplantation of teeth with complete rhizogenesis: a literature review. Revista Científica do CRO-RJ (Rio de Janeiro Dental Journal), 4(1), 10-15. https://doi.org/10.29327/24816.4.1-3

Duarte, É. E. N., Macedo, I. R. R., de Carvalho, W. R. S., do Couto Oliveira, H., Cristino, M. R., \& Neto, N. C. (2017). Autotransplante dentário-uma alternativa viável para a reabilitação dentária. Revista Digital APO, 1(1), 29-34. https://doi.org/10.5935/2526-8155.20170005

Giancristófaro, M., Júnior, W. P., Júnior, N. V. R., Júnior, H. M., \& de Oliveira, C. (2017). Transplante dental: revisão da literatura e relato de caso. Revista de Odontologia da Universidade Cidade de São Paulo, 21(1), 74-78. https://doi.org/10.26843/ro_unicid.v21i1.439

Jodas, C. R. P., Rapoport, A., Junqueira, J. L. C., Baccarin, L. S., Moraes, P. D. C., Gati, C. A. D. R., \& Teixeira, R. G. (2012). Transplante dental bilateral: relato de caso clínico com acompanhamento de 9 anos. Revista da Associacao Paulista de Cirurgioes Dentistas, 66(1), 30-35. http://revodonto.bvsalud.org/scielo.php?pid=S0004-52762012000100005\&script=sci_arttext

Kumar, R., Jyothi, M., Sirisha, K., Racca, K., \& Uma, C. (2012). Autotransplantation of mandibular third molar: a case report. Case reports in dentistry, 2012, 629180-629180. 10.1155/2012/629180

Mendes, K. D. S., Silveira, R. C. D. C. P., \& Galvão, C. M. (2008). Revisão integrativa: método de pesquisa para a incorporação de evidências na saúde e na enfermagem. Texto \& contexto-enfermagem, 17(4), 758-764. http://dx.doi.org/10.1590/S0104-07072008000400018

Mikami, J. R., Laureano Filho, J. R., Nogueira, P. T. B. D. C., Brasil Júnior, O., Silva Júnior, E. Z. D., \& Nogueira Filho, L. L. T. (2014). Transplante dental autógeno-Relato de caso. Revista de Cirurgia e Traumatologia Buco-maxilo-facial, 14(4), 51-58. http://revodonto.bvsalud.org/scielo.php?pid=S1808$52102014000400008 \&$ script=sci_arttext\&tlng=pt

Nimčenko, T., Omerca, G., Varinauskas, V., Bramanti, E., Signorino, F., \& Cicciù, M. (2013). Tooth auto-transplantation as na alternative treatment option: a literature review. Dental research journal, 10(1), 1. 10.4103/1735-3327.111756 
Research, Society and Development, v. 10, n. 3, e27410313331, 2021

(CC BY 4.0) | ISSN 2525-3409 | DOI: http://dx.doi.org/10.33448/rsd-v10i3.13331

Peixoto, A. C., Melo, A. R., \& Santos, T. D. S. (2013). Dental Transplantation: Literature Update and Case Report. Revista de Cirurgia e Traumatologia Bucomaxilo-facial, 13(2), 75-80. http://revodonto.bvsalud.org/scielo.php?pid=S1808-52102013000200012\&script=sci_abstract\&tlng=en

Pinto Júnior, A. A. C., Costa, S. M. A., Cunha, J. F. D., \& Palmier, A. C. (2018). Two-stage technique in third molar autotransplantation: case report. RGORevista Gaúcha de Odontologia, 66(1), 96-100. https://doi.org/10.1590/1981-863720180001000133388

Rebouças, D. S., Cerqueira, L. S., Andrade, E. L., Santos, H. L., Zerbinati, L. P. S., \& Assis, A. F. (2015). Autogenous tooth transplantation: Rehabilitative and viable option for SUS. Journal of Dentistry \& Public Health, 6(1). http://dx.doi.org/10.17267/2596-3368dentistry.v6i1.576

Santos, L. C. S., Dourado, V. C., Vieira, A. C., da Silva, C. A., \& Luz, M. D. N. (2013). Transplante dental autógeno como al ternativa para reabilitação oral: Relato de caso. Revista Uningá Review, 14(1), 10-10.

Stange, K. M., Lindsten, R., \& Bjerklin, K. Autotransplantation Of Premolars To The Maxillary incisor region: a long-term follow-up of 12-22 years. European journal of orthodontics, 38(5), 508-515, 2016. http://34.233.57.254/index.php/uningareviews/article/view/710

Tirali, R. E., Sar, C., Ates, U., Kizilkaya, M., \& Cehreli, S. B. (2013). Autotransplantation of a supernumerary tooth to replace a misaligned incisor with abnormal dimensions and morphology: 2-year follow-up. Case reports in dentistry, 2013. https://doi.org/10.1155/2013/146343

Warmeling, M., Estivalet, V. S., Conte, P. C., Jardim, L. T., Fritscher, G. G., \& Pagnoncelli, R. M. (2019). Transplante dentário autógeno: revisão de literatura e relato de caso clínico. Revista da Faculdade de Odontologia-UPF, 24(2), 273-278. https://doi.org/10.5335/rfo.v24i2.10449

Yoshino, K., Kariya, N., Namura, D., Noji, I., Mitsuhashi, K., Kimura, H., \& Matsukubo, T. (2012). Risk factors affecting third molar autotransplantation in males: a retrospective survey in dental clinics. Journal of oral rehabilitation, 39(11), 821-829. https://doi.org/10.1111/j.1365-2842.2012.02325.x 